\title{
Retos y perspectivas de la primera Estrategia europea de cultura
}

La Comisión Europea adoptó el pasado 10 de mayo una comunicación sobre la función de la cultura en el mundo globalizado, con la que se propone la creación de la primera estrategia europea para la cultura (http:// ec.europa.eu/culture/eac/communication/ comm_en.html). En la Comunicación se afirma el puesto central de la cultura en el proceso de integración europea y se propone una agenda cultural para Europa y para sus relaciones con los terceros países. La adopción de la Comunicación se produce tras un amplio proceso de consulta en el que participaron instancias decisorias y partes interesadas de toda Europa.

Los profesionales del sector reclamaban desde hace tiempo que la Unión Europea adoptara un papel más relevante en el ámbito de la cultura, que se pusieran las bases para una verdadera política cultural europea, por lo que en principio esta comunicación se ha recibido con optimismo. Sin embargo ahora queda por ver si esta estrategia contribuirá realmente a sus objetivos o se quedará en una simple declaración de intenciones, si conseguirá su propósito de hacer de la Unión Europea un proyecto social y cultural y no sólo económico y de integrar sistemáticamente la cultura en los programas y proyectos de desarrollo; es decir si se la dotará de medios para ello y si se acompañarán estos objetivos de políticas y acciones concretas.

En cuanto a sus objetivos la estrategia se apoya en tres pilares fundamentales: la promoción de la diversidad cultural y el diálogo intercultural, el fomento de la cultura como catalizador para la creatividad, el crecimiento y el empleo, y la promoción de la cultura como elemento vital para las relaciones internacionales de la Unión Europea. Si en el ámbito conceptual la estrategia destaca por la aportación positiva al debate internacio- nal sobre la protección y el fomento de la diversidad cultural junto al apoyo a la economía de las industrias creativas, a nivel operativo o instrumental podemos destacar el papel tan relevante que otorga a la cooperación cultural.

Partiendo del reconocimiento de la complejidad del sector cultural por su heterogeneidad y falta de estructuración, se plantean medidas concretas para hacer frente a esta situación buscando el diálogo y la participación del mayor número de agentes, nuevos métodos que faciliten la coordinación de los instrumentos existentes y una mayor presencia de lo cultural en las relaciones internacionales.

\section{Queda por ver si esta estrategia contribuirá} realmente a sus objetivos

\section{o se quedará en una simple declaración de intenciones}

Las medidas concretas que se proponen para la cooperación entre agentes y hacia el exterior de la Unión son:

$\rightarrow$ Promover el diálogo con el sector cultural: puesta en marcha de redes y plataformas que den mayor visibilidad al sector y faciliten la interlocución. Se propone entre otras cosas la realización de un inventario que permita conocer mejor la realidad y volumen del sector, así como la creación de un foro cultural que estimule la participación del conjunto de agentes.

$\rightarrow$ Creación de un método abierto de coordinación, MAC: este método se ofrece como un marco apropiado para la cooperación cultural entre los estados miembros, por ser un marco intergubernamental, no vinculante que consiste en acordar objetivos comunes, hacer un seguimiento periódico e intercambiar buenas prácticas.

$\rightarrow$ Conocer la contribución real del sector a los objetivos de la Agenda de Lisboa: apoyo a la creación y mantenimiento de sistemas estadísticos nacionales que permitan una mayor comparabilidad y difusión de los mismos.

$\rightarrow$ Integración de la cultura en el resto de políticas: el objetivo es mejorar la coordinación interservicios, incluyendo una especial atención en los programas de cooperación exterior a la diversidad y el diálogo intercultural.

Hasta el momento la enorme dispersión de los escasos fondos y programas de apoyo a la cultura no había contribuido en absoluto a la consolidación de un tejido cultural sólido y plural y las estructuras comunitarias no habían sido capaces de convertirse en un referente para los profesionales de la cultura, cansados de bucear de portal en portal para encontrar información oportuna de las convocatorias, cuando no de competir con cientos de aspirantes por la financiación de proyectos necesariamente pequeños y puntuales. Esperamos por tanto que esta comunicación abra realmente nuevas vías para la coordinación y armonización de políticas y que podamos dejar poco a poco de disfrazar los proyectos de cooperación cultural, y defender ante cualquier foro que la cultura es por derecho propio "un aspecto imprescindible para lograr los objetivos comunitarios estratégicos de prosperidad, solidaridad y seguridad."

\section{Pilar Tassara Andrade}

Empresa Pública de Gestión de Programas Culturales. Área de Cooperación 